Revista Voluntas: Estudos sobre Schopenhauer - Vol. 3, Números 1 e 2 - $1^{\text {o e }} 2^{\text {o }}$ semestres de 2012 - ISSN: 2179-3786 - pp. 61-74.

\title{
Nessun "accomodamento": Deussen e Hartmann contro l'eudemonologia di Schopenhauer*
}

\author{
Nenhuma "acomodação": Deussen e Hartmann contra a eudemonologia de \\ Schopenhauer
}

\author{
Giuseppe Invernizzi \\ Professor na Università degli Studi di Pavia (Pavia-Itália) \\ E-mail: giuseppe.invernizzi@unipv.it
}

\begin{abstract}
Riassunto: Questo saggio si propone di illustrare i motivi per cui tanto Paul Deussen quanto Eduard von Hartmann, che nella parte etica delle loro filosofie si rifanno sia pur criticamente a Schopenhauer, diano poco spazio ad una sezione importante dei Parerga e paralipomena, ovvero ai noti Aforismi sulla saggezza della vita.

Parole chiave: Eudemonologia schopenhaueriana; Deussen; Hartmann.

Resumo: Este artigo pretende ilustrar os motivos pelos quais tanto Paul Deussen quanto Eduard von Hartmann, que na esfera ética de suas filosofias remetem-se a Schopenhauer de forma crítica, tenham dado pouco espaço a uma importante seção dos Parerga e paralipomena, a saber, aos famosos Aforismos para a sabedoria de vida.
\end{abstract}

Palavras-chave: Eudemonologia schopenhaueriana; Deussen; Hartmann.

Gli Aforismi costituiscono senza dubbio la parte più popolare dei Parerga, come risulta anche dalle numerose edizioni separate in lingua tedesca e in altre lingue ${ }^{1}$. Si potrebbe pensare ad una scarsa considerazione complessiva di quest'opera di Schopenhauer, ma al riguardo non ci sono sicuri indizi. Per quanto risulta, solo in Deussen si trova un passo in cui - anche se per inciso - egli si rammarica del fatto che i Parerga siano "purtroppo la più letta" delle opere di Schopenhauer².

Questi scritti trovano la loro giustificazione in un'assunzione di fondo, ovvero che sia legittimo occuparsi della «saggezza del vivere», vale a dire «dell'arte di percorrere la vita nel modo quanto più possibilmente piacevole e felice» e abbandonare «il più alto punto di vista metafisico ed etico» (secondo cui una vita felice è impossibile, motivo per cui l'uomo dovrebbe negare la propria volontà di vivere). E' quell'«accomodamento» a cui ci si riferisce nel titolo e che è - almeno formalmente messo in discussione dallo stesso Schopenhauer, «in quanto esso rimane attaccato al punto di vista

\footnotetext{
1 *Questo testo è una rielaborazione della relazione Keine "Ackommodation": Deussen und Hartmann gegen Schopenhauers Eudämonologie, presentata al Convegno Le cœur et la tête. Philosophie, mœurs, sciences dans les Parerga et Paralipomena de Schopenhauer, Paris, 6-7 ottobre 2011. Cfr. A. Hübscher, Schopenhauer-Bibliographie, Frommann-Holzboog, Stuttgart-Bad Cannnstatt, 1981, pp. 45, 57 s., 63 s., 72, 81, 91.

${ }^{2}$ Cfr. P. Deussen, Bericht über Hegel und Schopenhauer, «Archiv für Geschichte der Philosophie», 3 (1890), p. 159.
} 
comune ed empirico, e ne mantiene gli errori» ${ }^{3}$. Ma tale accomodamento ha una sua profonda ragion d'essere nel sistema e nella concezione della morale di Schopenhauer.

Bisogna dunque considerare anzitutto «il più alto punto di vista metafisico ed etico». La negazione della volontà - come atto assolutamente gratuito - è, secondo Schopenhauer, una sorta di miracolo e non una conseguenza necessaria degli sforzi morali dell'uomo. E' noto che il manifestarsi della negazione della volontà viene paragonato in varie occasioni all'imperscrutabile irruzione della grazia divina ${ }^{4}$. Inoltre gli sforzi morali dipendono dalle tendenze originali e immutabili del carattere di ciascun essere umano, che nella maggior parte dei casi vede una preponderanza dell'egoismo, vale a dire dell'affermazione di sé5. In breve, secondo Schopenhauer, la maggior parte delle persone sono sempre alla (vana) ricerca della felicità, e questa è l'unica cosa che possono fare. Da tale punto di vista, l'edonismo (e l'egoismo) è ineludibile come l'ascetismo e gli eudemonisti non possono che pensare ed agire nei termini degli Aforismi. Va inoltre tenuto presente che in Schopenhauer, per quanto l'uomo buono si apra al prossimo e non percepisca più il mondo come un non-Io che gli è inevitabilmente ostile, la moralità non ha mai una dimensione autenticamente sociale e ancor meno politica: Schopenhauer è convinto che un reale progresso sia impossibile ${ }^{6}$.

Per quanto concerne il ruolo e l'importanza che Deussen ed Hartmann attribuiscono alla filosofia di Schopenhauer, entrambi perseguono un obiettivo comune, vale a dire dimostrare che Schopenhauer non rappresenta un momento di rottura all'interno della tradizione filosofica tedesca, ma al contrario si inserisce organicamente in essa, costituendone un momento importante se non decisivo. Per raggiungere questo obiettivo, entrambi procedono ad una sorta di "normalizzazione" di Schopenhauer.

$\mathrm{Al}$ riguardo è istruttivo un aneddoto. Deussen ottenne il suo primo grande successo accademico nel 1875/1876 con un corso tenuto ad Aquisgrana come Privatdozent. L'argomento delle lezioni fu essenzialmente la filosofia di Schopenhauer quale è esposta nel testo nato da tale corso, gli Elementi

\footnotetext{
${ }^{3}$ Cfr. A. Schopenhauer, Parerga und Paralipomena, in Sämtliche Werke, a cura di A. Hübscher, Brockhaus, Mannheim $1988^{4}$, vol. V, p. 331 [nel seguito P I], tr. it. di G. Colli, Adelphi, Milano 1981, p. 423: «Ich nehme den Begriff der Lebensweisheit hier gänzlich im immanenten Sinne, nämlich in dem der Kunst, das Leben möglichst angenehm und glücklich durchzuführen, die Anleitung zu welcher auch Eudämonologie genannt werden könnte. [...] Um eine solche dennoch ausarbeiten zu können, habe ich daher gänzlich abgehn müssen von dem höheren, metaphysisch-ethischen Standpunkte $\mathrm{zu}$ welchen meine eigentliche Philosophie hinleitet. Folglich beruht die ganze hier zu gebende Auseinandersetzung gewissermaßen auf einer Ackommodation, sofern sie nämlich auf dem gewöhnlichen, empirischen Standpunkt bleibt und dessen Irrthum festhält».

${ }^{4}$ Cfr. A. Schopenhauer, Die Welt als Wille und Vorstellung, in Sämtliche Werke, cit., vol. II, [nel seguito W I], p. 477 ss., tr. it. di S. Giametta, Rizzoli, Milano 2002, vol. I, p. 697 ss.

${ }^{5}$ Cfr. A. Schopenhauer, Die beiden Grundprobleme der Ethik, in Sämtliche Werke, cit., vol. IV, [nel seguito E], p. 252 ss., tr. it. di E. Pocar, Laterza, Bari 1970, p. 261 ss. A ciò si aggiunge che il carattere di ogni singolo uomo secondo Schopenhauer dovrebbe essere un'idea immutabile nel tempo. Questa dottrina non appare facilmente conciliabile con la teoria della ereditarietà del carattere, cui Schopenhauer si riferisce addirittura come all'unico mezzo per migliorare moralmente l'umanità (cfr. Die Welt als Wille und Vorstellung, In: Sämtliche Werke, cit., vol. III, [nel seguito W II], p. 604, tr. it. cit., vol. II, p. 739 s.

${ }^{6}$ Cfr. per es. W II, p. 507, tr. it. cit., p. 624 s.
} 
della metafisica ${ }^{7}$. Le sue prese di posizioni nei confronti della religione e soprattutto del cristianesimo furono interpretati come un episodio del Kulturkampf, e Deussen fu violentemente attaccato da un giornale reazionario locale, l'«Echo». Più tardi Burghard Freiherr von Schorlemer-Alst, deputato nel parlamento prussiano e avversario di Bismarck, riprese le critiche del giornale e - secondo il racconto di Deussen - sostenne in Parlamento che «C'è una regia università prussiana ... in cui un discepolo di Schopenhauer insegna che Cristo è uno sciocco (Dummkopf) $\rangle^{8}$. Qui si può sorvolare sugli ulteriori sviluppi della vicenda. Basti dire che nel 1878 il ministero impose a Deussen di «limitarsi alla storia della filosofia, cioè da Platone a Kant», vale a dire di non parlare di Schopenhauer ${ }^{9}$. Ciò che è interessante è che nel 1877 - vale a dire nel bel mezzo della polemica - Deussen ritenne opportuno inviare tre copie dei suoi Elementi di metafisica al principe ereditario. Due di queste copie avrebbero dovuto essere date ai figli di quindici e diciotto anni, se il principe ereditario avesse giudicato il libro adatto a due adolescenti. Secondo ogni evidenza Deussen era convinto che la filosofia di Schopenhauer - almeno nella versione degli Elementi - avrebbe potuto dare un contributo importante alla formazione di un futuro imperatore ${ }^{10}$.

Si è detto sopra che la "normalizzazione" di Schopenhauer si esprime nel tentativo di stabilire una connessione più solida tra Schopenhauer e la tradizione filosofica tedesca. Così Deussen sottolinea fortemente la continuità tra Kant e Schopenhauer. La teoria della conoscenza di Schopenhauer è una corretta interpretazione di quella di Kant e la sua metafisica non oltrepassa i confini che Kant aveva posto al sapere filosofico: l'intima natura della cosa in sé rimarrebbe anche per Schopenhauer indeterminata. Ma egli va oltre, e - come vedremo - pensa che anche la filosofia pratica di Kant concordi sostanzialmente con quella di Schopenhauer ${ }^{11}$.

L'inserimento di Schopenhauer nella tradizione viene portato avanti in grande stile da Hartmann ${ }^{12}$. In un lungo saggio, il cui titolo è Il triumvirato filosofico del diciannovesimo secolo,

\footnotetext{
${ }^{7}$ Cfr. P. Deussen, Die Elemente der Metaphysik. Als Leitfaden zum Gebrauche bei Vorlesungen sowie zum Selbststudium, Mayer, Aachen 1877; Brockhaus, Leipzig $1902^{3}$ [qui utilizzata]; tr. it. Gli elementi della metafisica, a cura di L. Suali, Mattei, Speroni \& C., Pavia 1912.

${ }^{8}$ Cfr. P. Deussen, Mein Leben, Brockhaus, Leipzig 1922, p. 183 s. Deussen precisa che egli non aveva mai usato tale espressione. Si trattava invece di una faziosa conclusione che il citato giornale aveva ricavato dal confronto istituito da Deussen fra le teologia indiana e quella cristiana circa il valore della legge e delle opere, in cui veniva affermata la superiorità del pensiero indiano (Cfr. Die Elemente der Metaphysik, cit., p. 240, tr. it. cit., p. 212).

${ }^{9}$ Cfr. Deussen, Mein Leben, cit., p. 186.

${ }^{10}$ Ibidem, p. 185. Sembra che effettivamente il libro sia stato dato al maggiore dei figli, ovvero a Wilhelm (che sarebbe divenuto imperatore), ma che questi non abbia trovato il testo particolarmente interessante, anche perché, su consiglio di Bona Meyer (da sempre acerrimo nemico di Schopenhauer), egli ne aveva letto una sprezzante recensione di L. Weis, in «Philosophische Monatshefte», 14 (1878), pp. 161-169.

${ }^{11}$ Cfr. Deussen, Die Elemente der Metaphysik, cit., p. 58, tr. it. cit., p. 68 s.

12 Sulla figura di Eduard von Hartmann (1842-1906) cfr. A. Drews, Eduard von Hartmanns philosophisches System in Grundriss, Winter, Heidelberg 1902, $1906^{2}$ e D. N. K. Darnoy, The unconscious and Eduard von Hartmann. A historico-critical monograph, Martinus Nijhoff, The Hague 1967. Per i suoi rapporti con Schopenhauer e il pessimismo mi permetto di rimandare anche a G. Invernizzi, Il pessimismo tedesco dell'Ottocento. Schopenhauer, Hartmann, Bahnsen e Mainländer e i loro avversari, La Nuova Italia, Firenze 1994.
} 
Schopenhauer è senza esitazione posto accanto a Schelling e Hegel, come una delle tre figure filosofiche più importanti del XIX secolo. La sua filosofia, interpretata senz'altro in senso speculativo, costituisce l'antitesi a quella di Hegel (il principio è la volontà invece della ragione), ma è anche come il necessario momento di transizione verso la filosofia positiva di Schelling, che Hartmann considera il diretto precorrimento del proprio sistema ${ }^{13}$.

E' appena il caso di ricordare quanto sia stato importante il ruolo Deussen nella storia della filosofia di Schopenhauer. Fu - come ha osservato Nietzsche - il primo degli Schopenhaueriani a ottenere una cattedra in Germania, fondò la "Schopenhauer Gesellschaft" e curò la migliore - anche se incompleta - edizione delle opere di Schopenhauer. Fu anche uno dei migliori indologi del suo tempo e le sue numerose traduzioni di testi indiani, insieme con la sua visione generale della filosofia indiana, hanno contribuito non poco alla diffusione del pensiero orientale in Occidente auspicata da Schopenhauer ${ }^{14}$.

La caratteristica più importante della sua interpretazione della filosofia di Schopenhauer è il rigore con cui egli fa valere in essa il precetto kantiano, secondo cui la conoscenza non può in nessun caso andare al di là della realtà fenomenica e può aver luogo solo nelle forme della nostra facoltà conoscitiva. Vi è pertanto un vasto settore della realtà che è e rimarrà per sempre inaccessibile all'uomo. Deussen ritiene tuttavia di poter comunque mantenere la tesi che la volontà è la cosa in sé, tenendo conto delle ben note considerazioni svolte al riguardo da Schopenhauer, secondo cui conosciamo la volontà, «solo in quanto si riflette nella forma di conoscenza del tempo, nel quale si manifesta come volere» ${ }^{15}$. Questo tuttavia non gli impedisce di inserire poco dopo un capitolo il cui titolo - identico a quello del venticinquesimo capitolo del secondo volume del Mondo - suona: «Considerazioni trascendenti intorno alla volontà come la cosa in sé». Anche il contenuto è essenzialmente la stesso: veniamo a sapere che la volontà è estranea ad ogni varietà e divisibilità, che è senza fondamento e quindi libera. Questa via negativa si trova già in Schopenhauer ed è irta delle difficoltà che sono stati evidenziate più volte ${ }^{16}$.

Nei paragrafi che seguono Deussen sviluppa ulteriormente tale linea di pensiero, sottolineando l'indipendenza dal tempo della volontà come cosa in sé. Anche questa tesi si trova già in

\footnotetext{
${ }^{13}$ Cfr. E. von Hartmann, Das philosophische Dreigestirn des neuzehnten Jahrhunderts, in Gesammelte Studien und Aufsätze gemeinverständlichen Inhalts, Duncker, Berlino 1876, pp. 549-729.

${ }^{14}$ Sulla figura di Paul Deussen (1845-1919) cfr. la biografia di H. Feldhoff, Nietzsches Freund. Die Lebensgeschichte des Paul Deussen, Böhlau, Köln-Weimar-Wien 2008. Per il suo ruolo all'interno della «Schopenhauer Gesellschaft», da lui fondata, cfr. in particolare F. Ciracì, In lotta per Schopenhauer. La «Schopenhauer-Gesellschaft» fra ricerca filosofica e manipolazione ideologica 1911-1948, Pensa Multimedia, Lecce, 2011. Cfr. in generale anche D. Fazio, La scuola di Schopenhauer. Testi e contesti, Pensa Multimedia, Lecce, 2009.

${ }^{15}$ Deussen, Die Elemente der Metaphysik, cit., p. 90, tr. it. cit., p. 95. Il testo tedesco dice: «... sofern er in der Erkenntnisform der Zeit sich abspiegelt, in welcher es als Wollen auseinandergezogen erscheint».

${ }^{16}$ Deussen, Die Elemente der Metaphysik, cit., pp. 106-110. tr. it. cit., pp. 108-111. Per Schopenhauer cfr. W II, p. 361, tr. it. cit., p. 449 [Transzendente Betrachtungen über den Willen als das Ding an sich].
} 
Schopenhauer, ma egli ne radicalizza la portata. In un ulteriore capitolo intitolato «Esposizione mitica dell'evoluzione del mondo», in cui vien fatto largo ricorso a citazioni tratte dalla sapienza indiana, Deussen spiega che il passaggio dalla affermazione alla negazione della volontà non solo non è un evento che accade nel tempo (cosa che già Schopenhauer aveva sostenuto ${ }^{17}$ ), ma che affermazione $\mathrm{e}$ negazione sono due modi di essere del volere sempre compresenti: «la caduta degli spiriti accadde in un imperscrutabile passato, e accade tuttavia ora e ad ogni momento. Ma anche la salutare negazione è sempre presente: e chiunque nega salva se stesso e con sé tutte le creature, perché egli è tutta quanta la volontà di vivere. [...] e pur tuttavia l'affermazione permane anche dopo che egli si è sottratto al suo dominio, e questo mondo continuerà per tutti i tempi ad esistere, ad affermare, a soffrire» ${ }^{18}$. L'affermazione della volontà non deve essere pensata come un evento che in qualche modo rovescia lo stato originale di negazione della volontà, né, simmetricamente, la negazione della volontà deve essere pensata come un evento che ripristina quello stato originale. Se è vero che la condizione originale della volontà è la negazione (Deussen dice: «il suo [=della volontà] vero stato è la negazione» ${ }^{19}$ ), è anche vero che l'affermazione è eternamente presente, e va intesa come «un'angoscia morbosa, una tendenza peccaminosa» che si è formata «non in un tempo determinato, ma in un'eternità di passato, oggi e sempre nei secoli futuri, come l'offuscarsi appena percepibile del chiarore del cielo» ${ }^{20}$. La realtà è quindi costituita da un'eterna coesistenza di questi due modi di essere della volontà.

Degno di nota è il fatto che venga respinta l'idea che la negazione della volontà possa condurre a un annichilimento totale della cosa in sé; anche se la filosofia può descrivere lo stato di negazione della volontà solo negativamente, Deussen ritiene che si tratti di una realtà positiva cui si può dare il nome di $\mathrm{Dio}^{21}$.

Sebbene Deussen neghi espressamente a tale realtà la personalità, a suo avviso il cristianesimo resta la migliore illustrazione popolare della vera filosofia. E dato che il popolo non può raggiungere il sapere filosofico, il cristianesimo deve essere conservato e diffuso - a quanto pare nella sua forma ortodossa $^{22}$. Si può essere sia un buon cristiano sia uno schopenhaueriano, come Deussen stesso aveva mostrato nella sua vita. Era nato nel villaggio di Oberdreis, dove suo padre era pastore. Avendo superato l'esame di teologia nel 1871, egli sostituiva il padre volentieri nel suo ufficio, quando tornava nel suo paese natale ${ }^{23}$.

\footnotetext{
${ }^{17}$ Cfr. A. Schopenhauer, Gesammelte Briefe, a cura di A. Hübscher, Bouvier, Bonn 1987, p. 214.

${ }^{18}$ Deussen, Die Elemente der Metaphysik, cit., p. 112, tr. it. cit., p. 112 [Mytische Darstellung des Weltprozesses].

${ }^{19}$ Ibidem, p. 112, tr. it. cit., p. 113.

${ }^{20}$ Ibidem, p. 110, tr. it. cit., p. 111. Il testo tedesco dice: «Da bildete sich, nicht zu irgend einer Zeit, sondern vor Ewigkeiten, heute und immmerfort, vergleichbar einer unbegreiflichen Trübung der Himmelsklahrheit, in der reinen, schmerzund wollelosen Seligkeit der Verneinung ein krankhafter Hang, eine sündliche Wendung: die Bejahung des Willens zu Leben».

${ }^{21}$ Ibidem, p. 267 s., tr. it. cit., p. 233.

${ }^{22}$ Ibidem, p. 208, tr. it. cit., p. 188.

${ }^{23}$ Cfr. Feldhoff, Nietzsches Freund, cit., p. 96.
} 
Deussen vede bene i limiti di questa posizione, tanto è vero che conclude la sua esposizione dicendo: "Queste sono le contraddizioni di cui appare piena la verità metafisica allorché noi vogliamo rivestirla di parole e di concetti propri del pensiero fisico» ${ }^{24}$. Ciò che è interessante per il tema di questo saggio è la trasformazione dell'etica di Schopenhauer che ne deriva.

Parlando dell'imperativo categorico, Deussen afferma che Kant, insieme a numerosi errori, ha sostenuto anche molte dottrine corrette. Ha anzitutto compreso - anche se non lo ha espresso chiaramente - che l'essenza della moralità consiste nella negazione del proprio sé ${ }^{25}$. Ha poi colto che l'imperativo è qualcosa di immediatamente presente in ogni essere umano, benché ne abbia posto erroneamente le radici nella ragione invece che nella volontà. Esso sarebbe dunque «un impulso a superare il nostro uomo naturale in quanto qualcosa che non deve essere, che ha le sue radici nelle profondità inconsce della cosa in sé e che da lì si manifesta nella coscienza ${ }^{26}$. Per Deussen la negazione dell'istinto egoistico non è altro che la negazione della volontà. Le azioni morali non sono momenti preparatori alla negazione del volere come voleva Schopenhauer ${ }^{27}$, ma la negazione stessa del volere. Da questo punto di vista la noluntas non è un qualcosa di ulteriore che interviene alla fine del processo ascetico, ma una condizione che si esprime positivamente e durevolmente nelle azioni morali $(\text { altruistiche })^{28}$.

Ma come è possibile sostenere che le azioni morali sono l'espressione e la realizzazione della negazione del volere, se ogni azione deriva sempre dalla volontà, vale a dire dalla sua affermazione? Deussen è ben consapevole che l'uomo conosce sempre e solo la volontà nella sua affermazione, e che tutto, nel mondo fenomenico e in lui, appare espressione di un tale volere. Pertanto qualsiasi atto è conosciuto sempre attraverso il principio di ragion sufficiente, vale a dire come conseguenza di un movente. E un motivo può operare solo in quanto in qualche modo prospetta una soddisfazione della volontà. Deussen ritiene di risolvere la questione in questo modo: ci sono due tipi di egoismo: un egoismo vero e proprio o egoismo individuale, in forza del quale noi siamo interessati solo al nostro benessere, e un egoismo apparente o morale, che «si estende al prossimo, a tutti gli uomini, infine, a tutte le creature esistenti». Questo egoismo rappresenta l'unica e imperfetta forma in cui si possono conoscere le autentiche azioni morali e la negazione della volontà ${ }^{29}$.

\footnotetext{
${ }^{24}$ Deussen, Die Elemente der Metaphysik, cit., p. 112, tr. it. cit., p. 113.

${ }^{25}$ Cfr. P. Deussen, Der kategorische Imperativ. Rede zur Feier des Geburtstages Sr. Majestät des deutschen Kaisers, Königs von Preußen, Wilhelm II., Kiel 1891, ripreso quasi integralmente in P. Deussen, Allgemeine Geschichte der Philosophie, vol. 2, parte 3 [Die neuere Philosophie von Descartes bis Schopenhauer], Brockhaus, Leipzig 1917, 1920², p. $259 \mathrm{~s}$.

${ }^{26}$ Deussen, Allgemeine Geschichte der Philosophie, cit., p. 268.

${ }^{27}$ Cfr. W II, p. 695, tr. it. cit., p. 850.

${ }^{28}$ Deussen, Die Elemente der Metaphysik, cit.,p. 247 s., tr. it. cit., p. 218.

${ }^{29}$ Ibidem, pp. 248-252, tr. it. cit., pp. 219-222.
} 
Ancora due osservazioni: secondo Deussen ogni uomo sente nella sua interiorità la voce dell'imperativo morale. In questo modo, la tendenza alla negazione non è più qualcosa di straordinario e di accessibile a poche persone: è universalmente presente e universalmente valida come universale è la voce della coscienza. L'uomo ha sempre la possibilità di agire moralmente e se non obbedisce alla voce della coscienza, egli agisce semplicemente in modo egoistico, ovvero immorale. E non è un caso che Deussen al paragrafo 269 dei suoi Elementi per rappresentare le tre sfere dell'egoismo, utilizza la tripartizione, con cui Schopenhauer inizia negli Aforismi l'analisi del concetto di felicità: che cosa è l'uomo, che cosa ha, che cosa rappresenta. Subito dopo, Deussen dice che bisognerebbe parlare piuttosto che di eudemonologia di egoismologia ${ }^{30}$.

Si è visto che secondo Deussen l'intenzione è fondamentale per la valutazione dell'atto morale e ciò concorda senz'altro con la filosofia pratica di Kant. In questo contesto tuttavia tale tesi assume un nuovo significato, nella misura in cui l'ascetismo appare essere l'unica intenzione moralmente legittima. Non importa quello che fa un uomo; importa che nel farlo rinneghi se stesso. Ne consegue che un perfetto discepolo di Schopenhauer può vivere una vita normale, svolgere qualsiasi professione: «perciò noi dobbiamo, non modificare la nostra attività, ma sibbene la disposizione d'animo da cui essa trae origine, e, invece di rifugiarci in un quietismo che aborre ogni fatica (nicht $z u$ müßsiggängerischem Quietismus), perseverare coraggiosamente nella lotta della vita, consci che la fatica dell'esistenza ci fu imposta per purificarci dall'egoismo e dalla colpa che da questo deriva» ${ }^{31}$. Questa indicazione concorda con i precetti evangelici ed anche con il pensiero indiano ${ }^{32}$. Quale modo migliore per neutralizzare gli aspetti più sovversivi della dottrina di Schopenhauer?

Sicuramente non si può sostenere che Eduard von Hartmann sia stato un discepolo ortodosso di Schopenhauer. Spesso egli ha tentato di minimizzare la componente schopenhaueriana del suo sistema e di rivendicare la sua vicinanza alla tradizione hegeliana. Non per caso tra i suoi ultimi lavori si trova un'estesa Dottrina delle categorie ${ }^{33}$. Ciò non ostante è stato uno degli scrittori che più hanno contribuito alla diffusione della filosofia di Schopenhauer, in particolare con la sua Filosofia dell'Inconscio, in cui la filosofia del maestro vien fatta dialogare con il positivismo e l'hegelismo. Nella prefazione alla decima edizione della sua opera più fortunata egli caratterizza in questo modo il suo sistema: «Esso è una sintesi di Hegel e di Schopenhauer con una decisa preponderanza del primo, portata a compimento secondo le indicazioni della dottrina dei principi della filosofia positiva di Schelling e del concetto di inconscio derivante dal primo sistema di Schelling; il risultato

\footnotetext{
${ }^{30}$ Ibidem, pp. 200-222, tr. it. cit., pp. 197-199.

${ }^{31}$ Ibidem, p. 259, tr. it. cit., p. 227. Si sa quanto frequentemente veniva stigmatizzato dagli avversari di Schopenahuer il quietismo cui avrebbe condotto la sua morale.

32 Deussen cita, fra gli altri, niente meno che il Codice di Manu: «L'ascesi dei dotti è la conoscenza, l'ascesi dei guerrieri è la difesa, l'ascesi degli operari è il lavoro, l'ascesi degli schiavi è il servire», Ibidem, p. 259, tr. it. cit., p. $226 \mathrm{~s}$.

${ }^{33}$ E. von Hartmann, Kategorienlehre, Haacke, Leipzig, 1896.
} 
provvisoriamente ancora astratto-monistico di questa sintesi è poi fuso con l'individualismo leibniziano e il realismo della scienza della natura moderna in modo da costituire un monismo concreto, in cui il pluralismo real-fenomenico è diventato un momento superato. Da ultimo il sistema risultante raggiunge la sua compiuta elaborazione a partire da una base empirica con il metodo induttivo delle moderne scienze naturali e storiche» ${ }^{34}$.

L'interpretazione di Hartmann della metafisica di Schopenhauer si differenzia da quella di Deussen in un punto fondamentale: tanto più Deussen cerca di mantenere separato il mondo fenomenico - accessibile all'uomo - dal mondo della cosa in sé - inconoscibile -, tanto più Hartmann sostiene la possibilità di una conoscenza della cosa in sé, del processo attraverso il quale l'Assoluto dà origine al mondo, seppure con la limitazione che questa conoscenza è solo probabile. Attraverso costruzioni non raramente audaci ed astratte, egli cerca di risolvere i problemi della filosofia di Schopenhauer con gli strumenti della speculazione ${ }^{35}$.

Schematicamente, la filosofia di Hartmann sostiene che all'origine del mondo c'è un Uno-Tutto - l'inconscio, unione di volontà e rappresentazione. Esso è costituito non solo dalla volontà ma anche dall'idea, che determina originariamente le direzioni del volere. L'atto della volontà attraverso il quale il mondo viene all'esistenza, è irrazionale, ma il contenuto di questo atto, nella misura in cui è condizionato dall'idea, è razionale e teleologicamente ordinato. Lo sviluppo del mondo ha una direzione e un fine: convincere la volontà che l'esistenza del mondo è un errore, vale a dire che la non-esistenza del mondo è preferibile alla sua esistenza, ovvero che il suo non essere è preferibile all'essere ${ }^{36}$.

Si vedrà in seguito per quali motivi il non-essere sia preferibile all'essere. Ora si devono considerare più nei dettagli i caratteri dell'interpretazione della metafisica di Schopenhauer sviluppata da Hartmann. Egli giudica ormai come acquisita l'opinione che «nell'intero sistema di Schopenhauer vi è una costante contraddizione tra la sua teoria della conoscenza idealistica e la sua metafisica che si fonda su premesse realistiche» ${ }^{37}$. Si è di conseguenza posti davanti all'alternativa di abbandonare o la sua teoria della conoscenza o la sua metafisica (e la sua etica). Poiché Hartmann preferisce salvare la

\footnotetext{
${ }^{34}$ E. von Hartmann, Philosophie des Unbewussten, Duncker, Berlin, 1869, Friedrich, Leipzig 1890 ${ }^{10}$, vol. I, p. XIII: «Dasselbe ist eine Synthese Hegel's und Schopenhauer's unter entschiedenem Uebergewicht des ersteren, vollzogen nach Anleitung der Principienlehre aus Schelling's positiver Philosophie und des Begriffs des Unbewussten aus Schelling's erstem System; das vorläufig noch abstract-monistische Ergebniss dieser Synthese ist alsdann mit dem Leibniz'schen Individualismus und dem modernen naturwissenschaftlichen Realismus zu einem concreten Monismus verschmolzen, in welchem der real-phänomenale Pluralismus zum aufgehobenen Moment geworden ist, und das so sich ergebende System ist endlich von empirischer Basis aus mit der inductiven Methode der modernen Natur- und Geschichtswissenschaften aufgebaut und errichtet».

${ }^{35}$ Cfr. Hartmann, Philosophie des Unbewussten, cit., vol. II, cap. XV [Die letzten Principien].

${ }^{36}$ Ibidem, vol. II, cap. XIII [Das Ziel des Weltprocesses und die Bedeutung des Bewusstseins].

${ }^{37}$ E. von Hartmann, Phänomenologie des sittlichen Bewußtseins, Duncker, Berlin 1879, Volksverband der Bucherfreunde, Berlin $1922^{3}$, p. 620.
} 
metafisica e l'etica, l'intera filosofia di Schopenhauer viene trasformata in senso realistico. L'Uno-Tutto (la volontà/cosa in sé di Schopenhauer) viene fatto convivere con la molteplicità non più solamente fenomenica degli individui e questa nuova filosofia viene chiamata monismo concreto. Hartmann spiega la sua teoria con queste parole: «l'individuo è un'oggettivazione reale ma transeunte e determinata dell'Uno-Tutto, la volontà di ciascuno è un fenomeno concreto della volontà del tutto nel momento della sua affermazione (la volontà di vivere). Non la sfera trascendente dell'unità, ma l'individuazione è il reale, per quanto non sia niente più che fenomeno dell'Uno che solo è

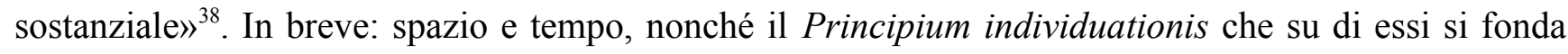
sono da considerarsi non più forme della facoltà conoscitiva umana, ma parti costitutive della struttura ontologica del mondo. Ne consegue che gli individui hanno una esistenza relativamente indipendente e che nel mondo vi è una vera storia, un vero sviluppo.

E' il momento di ritornare alla irrazionalità della realtà. Secondo Hartmann essa dipende dal fatto che i soggetti senzienti che esistono nel mondo nel loro complesso provano più dolore che piacere, come dovrebbe essere dimostrato dal celebre bilancio fra piacere e dolore

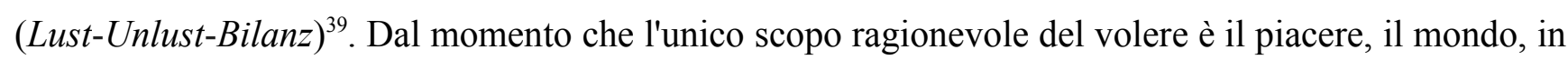
quanto non consente il raggiungimento di tale scopo, deve essere considerato irrazionale e in quanto tale non degno di esistenza. Hartmann non condivide con Deussen l'idea che sia possibile un nuovo e migliore stato del mondo - o dell'inconscio. L'unica soluzione possibile è il ritorno della volontà nello stato di non-volere, che significa l'annichilimento del mondo stesso ${ }^{40}$.

In questa prospettiva la questione della negazione della volontà assume un carattere del tutto nuovo. Poiché, come si è visto, gli individui sono da un punto di vista ontologico relativamente indipendenti, la negazione della volontà in un singolo individuo non presenta le difficoltà che si incontrano in Schopenhauer, secondo il quale l'individuo ha - almeno secondo la lettera del sistema solo realtà fenomenica. Questo ha però come conseguenze che la negazione del volere un individuo è quantitativamente irrilevante per il corso della storia - qualora si attribuisca un qualche significato allo sviluppo storico - e per il mondo nel suo complesso. Infatti, per ottenere l'annichilimento del mondo, è necessario che la maggior parte degli esseri senzienti e volenti (Hartmann si limita in pratica all'umanità) prenda la decisione di negare la propria volontà. Questo risultato metafísico è reso possibile dal fatto che il mondo non è altro che la somma delle volontà individuali: non vi è alcuna volontà trascendente, e le singole volontà costituiscono un individuo collettivo, che ha la capacità di

\footnotetext{
${ }^{38}$ Hartmann, Phänomenologie des sittlichen Bewußtseins, cit., p. 621.

${ }^{39}$ Come è noto, al calcolo di questo bilancio, la cui stessa possibilità è stata più volta messa in discussione dagli avversari del pessimismo, Hartmann dedica una estesa trattazione nella Philosophie des Unbewussten, cit., vol. II, pp. 285-355, cap. XIII [Die Unvernunft des Wollens und die Elend des Daseins]. Di questo capitolo e del seguente ho curato una traduzione italiana: Le illusioni del genere umano, a cura di G. Invernizzi, La scuola di Pitagora, Napoli, 2006.

${ }^{40}$ Cfr. Hartmann, Philosophie des Unbewussten, cit., vol. II, pp. 394-396.
} 
agire come un soggetto unitario. Un possibile conflitto fra tendenze diverse dovrebbe essere deciso dalla tendenza più forte, come avviene nella volontà di ogni singolo individuo. In questo caso, è la volontà della maggioranza a rappresentare la tendenza più forte ${ }^{41}$.

La negazione della volontà rimane lo scopo supremo dell'uomo, ma un tale scopo ha senso solo quando è portato a compimento per così dire al momento opportuno (cioè, solo se la maggioranza degli uomini decide di negare il volere nello stesso momento). In caso contrario, la negazione di se stessi non è altro che un atto egoistico, giacché chi lo compie non si preoccupa del bene complessivo dell'umanità e del mondo. Anche secondo Hartmann come per Deussen solo le azioni altruistiche hanno autentico valore morale. Pertanto, l'uomo agisce moralmente solo quando pone come suo fine il fine dell'umanità, vale a dire, il raggiungimento della beatitudine negativa del nulla. L'individuo, pur relativamente autonomo, è comunque parte del mondo e come tale ha la responsabilità del destino complessivo dell'umanità.

Si tratta quindi di convincere la maggioranza delle persone a negare la propria volontà. Per raggiungere questo obiettivo, ogni persona deve diffondere la conoscenza della irrazionalità dell'esistenza, ovvero della negatività del bilancio fra piacere e dolore. Ci sono tre illusioni che impediscono una chiara comprensione del risultato di tale bilancio e compito fondamentale è dunque la distruzione di tali illusioni: la prima illusione vorrebbe far credere che la felicità sia raggiungibile durante la vita terrena; mostrarne specificamente la falsità è compito del citato Lust-Unlust-Bilanz. La seconda illusione vorrebbe invece far credere che una vita felice dopo la morte (la felicità nell'aldilà) renderà positivo il bilancio fra piacere e dolore ${ }^{42}$. Secondo la terza illusione, la vita in una futura società riformata e avanzata dovrebbe garantire agli uomini un surplus di piacere ${ }^{43}$. A parere di Hartmann, le prime due illusioni sono già state praticamente distrutte. Che la vita terrena uomini offra più dolore che piacere, è ormai riconosciuto quasi da tutti. La credenza nell'immortalità dell'anima condizione della seconda illusione - di giorno in giorno è condivisa da un numero sempre più ridotto di persone. Resta solo la terza illusione, della cui vanità ci si potrà convincere solo quando la società del futuro che ne sta alla base sarà realizzata. In questa nuova società gli uomini potranno constatare che, anche in queste nuove condizioni, la felicità è impossibile. L'impossibilità di rendere positivo il bilancio piacere-dolore sarà allora chiara a tutti, e gli uomini potranno decidere di negare la volontà individuale, e quindi di annichilire il mondo.

Lo svelamento della terza illusione è quindi un compito pratico che si può realizzare solo attraverso la realizzazione di una società migliore. Hartmann non ritiene che la situazione dell'umanità in futuro possa davvero essere migliore di quella di oggi da un punto di vista eudemonologico. Crede

\footnotetext{
${ }^{41}$ Ibidem, pp. 396-404.

${ }^{42}$ Ibidem, pp. 355-368.

${ }^{43}$ Ibidem, pp. 368-390.
} 
tuttavia al progresso come mezzo per diffondere la coscienza della ineludibile sofferenza della vita. Tuttavia egli è consapevole che la piena realizzazione di tale progresso è un obiettivo che potrà essere raggiunto solo in un lontano futuro. Conseguentemente altrettanto si deve dire dello scopo finale della negazione della volontà. Tale obiettivo anzi finisce per essere così lontano da trovarsi praticamente al di fuori del campo visivo dell'uomo: ciò che è all'ordine del giorno, è il dovere di promuovere il progresso per costruire la nuova società. Pertanto, Hartmann non si stanca mai di spronare gli uomini a contribuire con coraggio e abnegazione al progresso economico e sociale ${ }^{44}$. In questo modo viene delineato un quadro completamente nuovo dei pessimismo e dei compiti dei pessimisti: il pessimista non è più un uomo che preferisce vivere in solitudine, indifferente alle vicende della storia, ai conflitti sociali, ai compiti cui si trova di fronte il suo paese. Il famoso quietismo di Schopenhauer non trova posto alcuno in questa nuova versione del pessimismo.

Hartmann cerca di confermare la verità della sua filosofia, mostrando che in Schopenhauer l'etica è in contraddizione con la dottrina della salvezza, ovvero con la negazione del volere. E' ben noto che secondo Schopenhauer sono due le vie che possono condurre l'individuo a tale passo: la conoscenza della sofferenza degli altri e l'esperienza diretta della propria sofferenza ${ }^{45}$. La seconda via è relativamente più comune, ed è il punto di partenza della critica di Hartmann. Se infatti la sofferenza è ingrediente necessario per giungere alla noluntas, ogni comportamento che si ponga il fine di alleviare il dolore degli uomini deve essere considerato come un passo nella direzione sbagliata. Ancor di più: «secondo il principio in base al quale bisogna fare agli altri quello che desidero che gli altri facciano a me, mi devo ritenere obbligato a infliggere agli altri la maggior quantità possibile di male $\mathrm{e}$ di sofferenza, che, se fosse inflitta a me, considererei come la suprema benedizione metafisica». Paradossalmente un despota orientale, che opprimesse e torturasse tutti i suoi sudditi con ogni mezzo, sarebbe un discepolo esemplare di Schopenhauer. Per evitare queste assurdità, è necessario sostituire la negazione della volontà individuale con la negazione della volontà generale e di conseguenza correggere radicalmente l'etica: come si è visto vi è un dovere verso l'umanità e tale dovere consiste nel favorire con ogni mezzo il progresso ${ }^{46}$.

Le modifiche apportate alla filosofia morale di Schopenhauer conducono a risultati che in definitiva si avvicinano alle posizioni di Deussen: anche per Hartmann la negazione della volontà non è un evento straordinario che riguarda solo pochi santi e asceti, ma un fine cui tutta l'umanità può sperare di raggiungere. Poiché tuttavia la sua concreta realizzazione è spostato in un lontano futuro,

\footnotetext{
${ }^{44}$ Ibidem, p. 404. Cfr. anche p. 531 s. Qui Hartmann precisa che, al limite, la dottrina della negazione finale della volontà e dell'annichilimento del mondo potrebbe essere lasciata cadere senza modificare il fine pratico dell'impegno sociale dell'individuo.

${ }^{45}$ Cfr. W I, pp. 446-471, tr. it. cit., pp. 660-689.

${ }^{46}$ Hartmann, Phänomenologie des sittlichen Bewußtseins, cit., p. 56.
} 
anche la morale di Hartmann prescrive all'uomo di impegnarsi altruisticamente nella vita quotidiana come vuole quella di Deussen. Di nuovo Schopenhauer è «normalizzato».

Per concludere, un cenno alle critiche rivolte da Hartmann alla tesi schopenhaueriana secondo cui la compassione è il fondamento della morale. Hartmann osserva in primo luogo che anche le azioni che derivano dalla compassione devono necessariamente avere una relazione con il benessere del soggetto che agisce, cosicché non possono che essere considerate egoistiche. Inoltre, anche azioni non suggerite dalla compassione possono essere morali ${ }^{47}$. Ne consegue che la compassione non può essere considerato l'unico fondamento della morale. Va anche considerato che la compassione, in quanto si basa sulla sensibilità, non è un criterio affidabile per determinare l'obiettivo valore morale di un atto ${ }^{48}$. Hartmann illustra con molti esempi come la compassione possa essere fuorviante e quanto spesso si corra il rischio, a causa della compassione, di fare la scelte morali sbagliate. Per esempio, un mendicante esperto può guadagnare molto di più di un vero povero che, per la vergogna, non osa chiedere con insistenza l'elemosina. La posizione di Schopenhauer deve essere intesa come una reazione contro l'unilaterale razionalismo Kant, ma ha anche un profondo fondamento nella sua metafisica $^{49}$. E in questo contesto Hartmann critica di nuovo Schopenhauer per l'esclusione di qualsiasi componente razionale dall'Uno-Tutto. Se la ragione non ha alcuna parte nella struttura ontologica del mondo, è impossibile che essa svolga un ruolo significativo nell'agire morale degli uomini. Il mondo in Schopenhauer non ha uno scopo razionale, e «noi chiamiamo razionale solo un agire finalizzato a scopi razionali $»^{50}$. Di conseguenza la ragione svolge un ruolo solo all'interno della cosiddetta saggezza morale, all'interno della quale «è la volontà egoistica, che cerca la felicità, quella che stabilisce i fini dell'azione e l'intelletto è solo il servo, che porta la lanterna affinché la volontà trovi con maggiore sicurezza la sua via ${ }^{51}$. Non è azzardato vedere in queste parole un'allusione al concetto di saggezza, che si trova all'inizio degli Aforismi di Schopenhauer. Anche Hartmann non ritiene possibile dare spazio all'eudemonologia dei Parerga.

\section{Nota bibliografica}

CIRACÌ, Fabio. In lotta per Schopenhauer. La «Schopenhauer-Gesellschaft» fra ricerca filosofica e manipolazione ideologica 1911-1948. Lecce: Pensa Multimedia, 2011 (Schopenhaueriana, 6).

DARNOY, D. N. K. The unconscious and Eduard von Hartmann. A historico-critical monograph, Martinus Nijhoff, The Hague 1967.

\footnotetext{
${ }^{47}$ Ibidem, p. 198 s.

${ }^{48}$ Ibidem, p. 200.

${ }^{49}$ Ibidem, p. 206.

${ }^{50}$ Ibidem, p. 275.

${ }^{51}$ Ivi.
} 
DEUSSEN, Paul. Die Elemente der Metaphysik. Als Leitfaden zum Gebrauche bei Vorlesungen sowie zum Selbststudium. Aachen: Mayer, 1877; Leipzig: Brockhaus, 1902.

. Gli elementi della metafisica. A cura di L. Suali, Mattei, Speroni \& C., Pavia, 1912.

. Mein Leben. Leipzig: Brockhaus, 1922.

. Allgemeine Geschichte der Philosophie, vol. 2, parte 3 [Die neuere Philosophie von Descartes bis Schopenhauer]. Leipzig: Brockhaus, 1917, $1920^{2}$.

. Der kategorische Imperativ. Rede zur Feier des Geburtstages Sr. Majestät des deutschen Kaisers, Königs von Preußen, Wilhelm II., Kiel, 1891.

Vol. 3 (1890).

. Bericht über Hegel und Schopenhauer, «Archiv für Geschichte der Philosophie»,

DREWS, A. Eduard von Hartmanns philosophisches System in Grundriss. Heidelberg: Winter, 1902 e 1906.

FAZIO, Domenico. M. La scuola di Schopenhauer. Testi e contesti. Lecce: Pensa Multimedia, 2009 (Schopenhaueriana, 2).

FELDHOFF, H. Nietzsches Freund. Die Lebensgeschichte des Paul Deussen, Böhlau, Köln-Weimar-Wien 2008.

HARTMANN, Eduard von. Philosophie des Unbewussten. Berlin: Duncker, 1869; Leipzig: Friedrich, 189010.

- Das philosophische Dreigestirn des neuzehnten Jahrhunderts, In: "Gesammelte Studien und Aufsätze gemeinverständlichen Inhalts". Berlin: Duncker, 1876.

. Kategorienlehre. Leipzig: Haacke, 1896.

. Phänomenologie des sittlichen Bewußtseins. Berlin: Duncker, 1879 (Volksverband der Bucherfreunde, Berlin 19223).

Pitagora, 2006.

. Le illusioni del genere umano. A cura di G. Invernizzi. Napoli: La scuola di

HÜBSCHER, Arthur. Schopenhauer-Bibliographie. Stuttgart-Bad Cannnstatt: Frommann-Holzboog, 1981. 
INVERNIZZI, Giuseppe. Il pessimismo tedesco dell'Ottocento. Schopenhauer, Hartmann, Bahnsen e Mainländer e i loro avversari. Firenze: La Nuova Italia, 1994.

SCHOPENHAUER, Arthur. Sämtliche Werke. Hrsg. von A. Hübscher. Mannheim: Brockhaus, 1988. . Gesammelte Briefe. Hrsg. von A. Hübscher. Bonn: Bouvier, 1987. . Parerga e paralipomena. Trad. it. G. Colli. Milano: Adelphi, 1981. . Il mondo come volontà e rapprezentazione (I e II). Trad. it. S. Giametta.

Milano: Rizzoli, 2002.

Recebido: 11/12/12

Received: $12 / 11 / 12$

Aprovado: 21/12/12

Approved: $12 / 21 / 12$ 